\title{
Urine and Kidney Epidermal Growth Factor: Ontogeny and Sex Difference in the Mouse
}

\author{
J. PERHEENTUPA, J. LAKSHMANAN, AND D. A. FISHER
}

Perinatal Research Laboratories, Harbor-UCLA Medical Center, Torrance, California 90509

\begin{abstract}
We have explored the physiology of urinary epidermal growth factor (EGF) in the mouse by studying its ontogeny, and the effects of testosterone therapy on immunoreactive EGF (IR-EGF) concentrations in urine, kidney, serum and submandibular gland (SMG). Urine IREGF (U-EGF) increased about 100 -fold relative to urea concentration and about 1000 -fold relative to urine volume from the 1st day of life to adulthood. Most of this increase occurred between days 6 and 18, which is the known period of steep rise in plasma thyroid hormone concentration in the mouse. A small $F>M$ sex difference was present in the adult. This difference was opposite in direction to the large $M>F$ sex difference in adult SMG-EGF concentration. On day 26 (age of appearance of morphological sex difference in SMG) the sex difference was not yet present in urine, although in SMG it was even larger than in the adult. Kidney EGF concentration was low relative to $U$ EGF: $1 \mathrm{ml}$ of adult urine contained approximately as much IR-EGF as 100 pairs of adult kidneys. In the adult, but not on day 26, there was a sex difference in kidney EGF concentration parallel to the sex difference in urine: female levels were about $30 \%$ higher than male levels. Ten days of testosterone treatment of adult female mice evoked an increase in IR-EGF concentration which was 1.7-fold for serum and 5-fold for SMG. In contrast, this treatment did not increase kidney or urine IR-EGF concentrations although kidney weight increased 1.3-fold. Our findings suggest that U-EGF originates from sources other than the SMG or blood. Rapid synthesis and secretion by the kidney may occur. Thyroid hormone appears to be a major regulator of U-EGF, but other factors also seem to be involved. (Pediatr Res 19: 428-432, 1985)
\end{abstract}

\section{Abbreviations}

EGF, epidermal growth factor

IR-EGF, immunoreactive EGF

U-EGF, urine IR-EGF

SMG, submandibular salivary gland

RIA, radioimmunoassay

EGF, a 6045 molecular weight polypeptide (1), is a potent mitogen for both epidermal and nonepidermal cells (2). It was discovered in mouse SMG (3), where it is present in extraordinarily high concentrations. EGF is synthesized and stored in the convoluted granular tubules of $\operatorname{SMG}(4,5)$. SMG-EGF is in-

Received June 21, 1984; accepted December 21, 1984.

Reprint requests: Dr. J. Perheentupa, Children's Hospital, SF-00290, Helsinki, Finland.

Supported by NIH Grants HD-04270 (D.A.F.) and NS-17431A (J.L.). creased by treatment with thyroxine or testosterone (6-8). Other tissues also contain IR-EGF, but in lesser and variable concentrations $(9,10)$. Among body fluids, urine contains the highest concentrations of IR-EGF (11), followed by milk (11), saliva (11), and bile (12). The concentration in blood plasma is low $(11,12)$.

The messenger RNA for prepro-EGF in mouse SMG contains seven EGF-like sequences (13). Very little is known of the origin, molecular nature, hormonal modulation, and physiological role of EGF in tissues other than SMG. Moreover, the relationships of the EGF content of tissues and body fluids are unknown.

Herein we report the concentration of mouse U-EGF during postnatal development. We also compare levels in males and females at a time when morphological sexual dimorphism develops in SMG with the appearance of convuluted granular tubules characteristic of the adult male (14). In addition, we examine the effects of testosterone on IR-EGF levels in SMG, kidney, serum, and urine. Our results suggest that 1) there is a progressive increase in U-EGF during normal development, 2) U-EGF levels do not change with development of a sexual dimorphism in SMG-EGF concentrations; 3) levels of IR-EGF in kidney and urine, in contrast to SMG, are higher in adult females than in adult males; and 4) levels of IR-EGF are testosterone sensitive in SMG and serum but not in kidney and urine.

\section{MATERIALS AND METHODS}

Animals and treatments. Swiss-Webster mice were used throughout; they were purchased from Simonsen Laboratories, Gilroy, CA. For studies of newborn, multiparous pregnant mice were received in our animal colony 4-5 days prior to parturition. They were immediately housed individually, kept under controlled temperature $\left(20^{\circ} \mathrm{C}\right)$ and lighting $(14 \mathrm{~h}$ light $)$ and provided with regular laboratory food and water ad libitum. The day of birth is considered day 0 . For studies at ages 12 and $24 \mathrm{~h}$, litter size was limited immediately after delivery to eight pups. For studies at ages 2-6 days, pups born within $4 \mathrm{~h}$ of one another were pooled and distributed eight pups per mother. For studies at ages 7-18 days, pups born within 14-16 h of one another were pooled and distributed eight pups per mother. For studies of 26day-old (age of appearance of morphological sex differences in SMG) postweanling mice, animals were purchased 4 days prior to sacrifice. Both sexes were housed separately, four animals per cage. Adult ( 3 to 4 month old) female and male mice were housed one to three per cage for at least 7 days prior to sacrifice. For testosterone response studies adult female mice were housed two per cage, injected subcutaneously every $48 \mathrm{~h} \times 5$ with equal volumes of sesame oil with or without testosterone propionate (12.5 or $25 \mu \mathrm{g} / \mathrm{g}$ body weight) and sacrificed on the day after the last injection. Animals were sacrificed by $\mathrm{CO}_{2}$ inhalation.

Handling and assay of samples. Urine was obtained by spontaneous voiding during handling of mice. Samples were collected individually and stored at $-20^{\circ} \mathrm{C}$. Blood was drawn from the 
abdominal vena cava, left at room temperature for $30 \mathrm{~min}$, and then transferred to an ice water bath. After 5-60 min in the bath, samples were centrifuged $(5 \mathrm{~min}$ at $9000 \times \mathrm{g})$ in a Beckman microfuge $B$ and serum immediately separated and stored at $-20^{\circ} \mathrm{C}$. Amniotic fluid was obtained by puncture of the amniotic cavity; samples were stored at $-20^{\circ} \mathrm{C}$. SMG and kidneys were removed, cleaned of adhering tissue, weighed, frozen on dry ice, and stored at $-70^{\circ} \mathrm{C}$. Both tissues were homogenized in phosphate-buffered saline, pH 7.2 at $4^{\circ} \mathrm{C}$, using a glass-Teflon homogenizer. The homogenates were centrifuged at $100,000 \times g$ for $30 \mathrm{~min}$ at $4^{\circ} \mathrm{C}$, and the supernatants used for measurements of protein (15) and EGF.

Upon thawing urine samples were centrifuged, and aliquots of clear urine were diluted in RIA buffer for determinations of ureaN (16) and EGF.

EGF was measured in urine, serum, amniotic fluid, kidneys, and SMG using a specific double antibody liquid phase mouse EGF RIA $(8,12,17,18)$.

EGF was purified from adult male Swiss Webster mice by the method of Savage and Cohen (19). Our purified EGF runs as a single band on polyacrylamide gel electrophoresis and manifests an apparent molecular weight of $6000 \mathrm{~d}$. Daily injections of $2 \mu \mathrm{g}$ $\mathrm{EGF} / \mathrm{g}$ body weight/day accelerates eyelid opening in newborn mouse pups from $13-14$ to 8-9 days $(8,17)$. This material is used for radioiodination and reference standard in the RIA systems. The EGF-RIA systems are sensitive to $15-20$ pg EGF $(6000 \mathrm{~d})$ and cross-react with the 74,000 d SMG storage form of the growth factor. The antiserum was used at a final dilution of $1: 250,000$. Cross-reaction with NGF is less than $0.01 \%$. There is no cross-reaction with SMG renin, insulin, human IGF-I, rat multiplication-stimulating activity, myelin basic protein, or cytochrome C. Intra- and interassay coefficients of variation are in the $5-10 \%$ range. Nonspecific binding consistently is less than $5 \%$.

Data analysis. Student's $t$ test was used to assess differences between group means: a $p<0.05$ value was considered significant. Tissue EGF concentrations were expressed per milligram protein. The distributions within the groups of EGF concentrations in urine and tissues were positively skewed. Therefore, all data were $\log$ transformed for calculations, and geometric means and mean $\pm S E$ intervals are presented throughout.

\section{RESULTS}

Serial dilutions of adult mouse urine and kidney homogenates produced RIA displacement lines parallel to purified EGF standard (17) (Fig. 1). When standard EGF in amounts varying from 50-500 pg was added to identical aliquots of a urine dilution, $84-118 \%$ (mean 105) were recovered in the RIA. Similarly, the recoveries of standard EGF added to kidney homogenate ranged from $92-112 \%$ (mean $103 \%$ ). The coefficients of interassay and intraassay variation were 4.2 and $2.3 \%$ for U-EGF, and 14 and $6.7 \%$ for serum EGF; values for kidney EGF were intermediate between those of urine and serum EGF.

In an amniotic fluid sample obtained on day 18 of gestation the EGF concentration was $290 \mathrm{pg} / \mathrm{ml}$. That is less than $10 \%$ of the average concentration in the urine on the 1st day of life. The simultaneously measured maternal serum IR-EGF concentration was $180 \mathrm{pg} / \mathrm{ml}$.

The postnatal ontogenic profile of U-EGF concentration (ng $\mathrm{EGF} / \mathrm{mg}$ urea-N) as a function of age included three distinct phases (Fig. 2). First, the concentration remained unchanged from day 1 through 6 . Second, a marked increase occurred by day 7 and continued through a plateau observed from day 12; this plateau level was some 20 -fold higher than the first plateau. Third, a further 2-fold increase in U-EGF appeared between days 15 and 18 with a plateau lasting until day 26 (not shown). Urine EGF levels of fully mature adults were still higher. Urine urea- $N$ concentrations (relative to adult levels) were low on the first 2 days of life and intermediate thereafter (Fig. 3). The increase in urea- $\mathrm{N}$ during the first 18 days was 5 - to 8 -fold compared to an 80- to 100 -fold increase in urine EGF concentration.
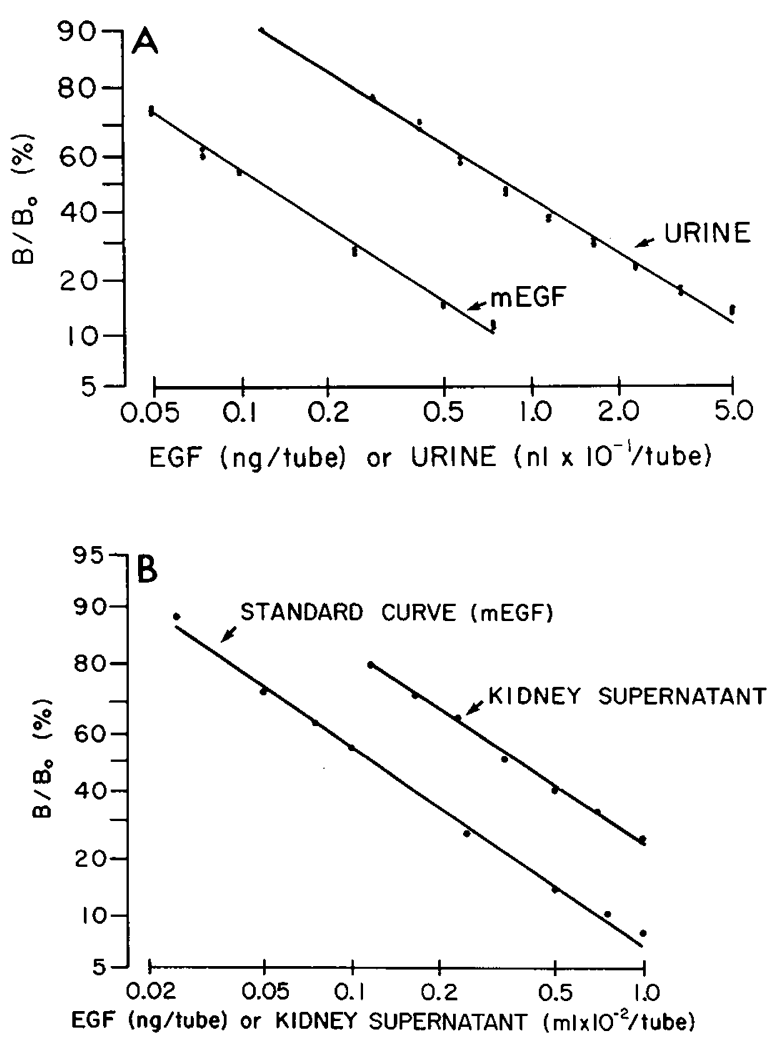

Fig. 1. Parallel diplacement in mouse EGF-RIA of ${ }^{125}$ I-EGF by serial dilution of standard mouse EGF and adult mouse urine $(A)$, and kidney homogenate $(B)$.

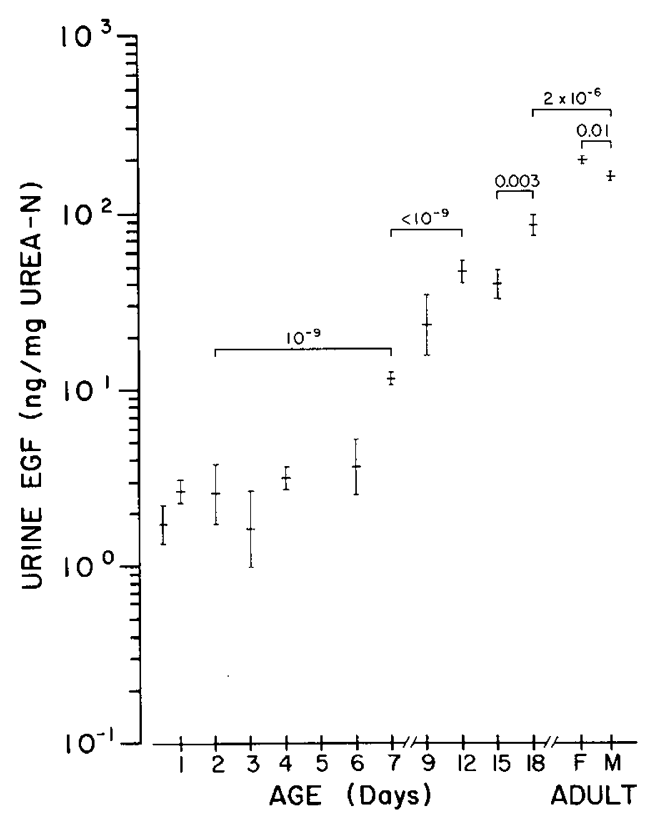

Fig. 2. Mouse urine immunoreactive EGF concentrations (mean \pm $\mathrm{SE}$ ) relative to urea concentration from the 1st day of life through day $18(n=3$ to 20$)$, and in adult females $(n=69)$ and males $(n=39)$. Exact $p$ values are given for significant differences between the two means under the ends of the parentheses.

SMG, kidney, and urine IR-EGF levels were compared in 26day-old and adult males and females. On day 26 the mean SMGIR-EGF concentration was 14-fold higher in males than females (Fig. $4 A$ ). In contrast, neither kidney (Fig. $4 B$ ) nor urine IR-EGF (Fig. $4 C$ ) concentrations exhibited a sex difference at this age. In 


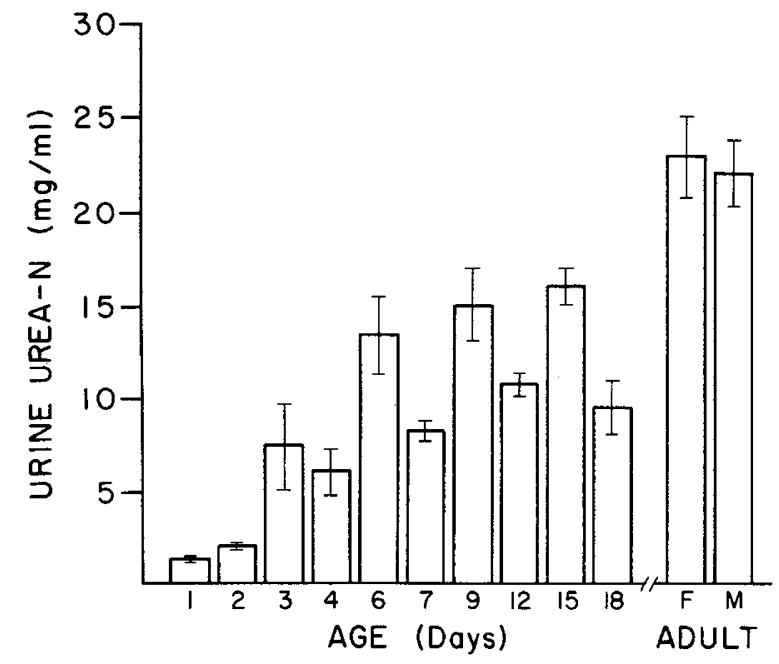

Fig. 3. Mouse urine urea-N concentrations (mean \pm SE) from the 1st day of life through day $18(n=3-12)$ and in adult females $(n=29)$ and males $(n=20)$.

fully mature adult mice the mean SMG-IR-EGF levels were higher than on day 26 by a factor of 60 in males and 150 in females (Fig. 4A). The adult SMG-IR-EGF levels were 10-fold higher in the male than the female. The relative kidney IR-EGF concentrations (Fig. $4 B$ ) were higher in the adult than 26-day animals by a factor of 2 in males and 4 in females. Similarly, UEGF concentration (Fig. $4 C$ ) was 2 -fold higher in the adults than in 26-day animals in each sex. However, in contrast to the SMG, IR-EGF concentrations in adult kidneys and urine were higher in females than in males. The difference was about $30 \%$ for the kidney and about $20 \%$ for urine. Moreover, mean urine IR-EGF levels in adult animals (ng/mg urea-N, Fig. $4 C$ ) were some 1000 fold higher than mean kidney levels (pg/mg, Fig. $4 B$ ). In fact 100 pairs of adult kidneys would contain the same amount of IR-EGF as $1 \mathrm{ml}$ of adult urine.

The relative mean kidney weights, in $\mathrm{g} / 100 \mathrm{~g}$ body weight, for males and females, respectively, were $1.55 \pm 0.04$ and $1.65 \pm$ 0.09 on day 26 and $2.04 \pm 0.06$ and $1.42 \pm 0.04$ in adults. The adult male value differed from the 26-day male level $(p=0.002)$ and from the adult female value $(p<0.001)$. Similarly, the relative male and female SMG weights, in $\mathrm{g} / 100 \mathrm{~g}$ body weight, were $0.706 \pm 0.023$ and $0.678 \pm 0.029$ on day 26 and $0.865 \pm$ 0.037 and 0.756 and 0.047 in adults; all these values were statistically similar.

The effects of testosterone treatment on serum, urine, kidney, and SMG IR-EGF levels of adult female mice are shown in Figure 5. Two testosterone doses were used: 12.5 and $25 \mu \mathrm{g} / \mathrm{g}$ body weight. The results obtained with the two doses were not different for any variable and were thus pooled. A 1.7-fold increase in serum IR-EGF and a 5-fold increase in SMG-IREGF were observed. In contrast, kidney and urine IR-EGF concentrations were unresponsive to testosterone. This was true despite the fact that the mean kidney weight increased by $34 \%$ $(p<0.001)$ and the mean SMG weight by $14 \%(p=0.005)$.

\section{DISCUSSION}

The present results provide data regarding concentrations of IR-EGF in neonatal mouse urine throughout the suckling period. U-EGF began to increase progressively after day 6 . There was the suggestion of a two-phase increase; one between days 6 and 12 and another between days 15 and 18 (Fig. 2). Compared to the logarithmic increase in U-EGF concentrations after day 6 , the 2- to 3-fold increase in urine urea (per milliliter) was small (Fig. 2). Expressing U-EGF concentrations per milliliter of urine rather than per milligram urea- $\mathrm{N}$ would give an even larger increase with age. However, we regard the concentration relative to urea as more meaningful because it eliminates the effect of variability in urine concentration. Although the molecular nature of U-EGF has not been investigated during early development, our recent studies (18) indicate that IR-EGF in mouse urine on day 7 is similar to the EGF standard (molecular weight 6045).

The factors controlling the ontogenic pattern of urine EGF are not entirely clear. Since urine EGF increases in response to thyroxine (18) and endogenous thyroid hormone levels increase steeply between 5 and 15 days (20), the increasing endogenous thyroid hormone levels may be responsible for the massive increase in urine EGF between days 6 and 18. Exogenous growth hormone also increases urine EGF during the 1st wk of life (21), and since thyroid hormones are known to stimulate growth hormone secretion, growth hormone may contribute to this ontogenic urine EGF surge. It is not clear whether the doubling of urine EGF levels between 18 days and adulthood is hormonally mediated. Testosterone treatment in the present studies has no effect on urine EGF. Uterine and kidney EGF in immature mice are sensitive to estradiol (22) and kidney and urine EGF levels were greater in female than in male adult animals in the present study. Thus it is possible that estradiol influences urine EGF in adult animals. In humans, high levels of U-EGF have been reported in females taking oral contraceptives (23).

The tissue origin of U-EGF during the early neonatal period is not entirely clear. Thyroxine and growth hormone increase UEGF during the 1st wk without significantly altering SMG or kidney EGF concentrations $(18,21)$. Thyroxine treatment also increases skin EGF levels (17) but serum levels are decreased (12). Thus, during the 1 st wk of life in the mouse, U-EGF levels seem to be regulated independently of tissue and serum EGF concentrations, and increased U-EGF levels are not associated with increased kidney EGF concentrations. In the adult animals in the present study testosterone increased SMG and serum EGF concentrations but had no effect on U-EGF. In addition, we observed a reverse sex difference in EGF concentrations in urine and kidney compared with SMG in the adult animals.

These observations suggest that U-EGF is not derived predominantly from the IR-EGF of SMG or blood. It could be generated by the kidneys from a nonimmunoactive precursor molecule in plasma but more likely it is produced by the kidney. This view is supported by the recent demonstration of EGF mRNA in the mouse kidney (24). EGF synthesized in kidney tissue might be stored in a nonimmunoreactive form in the kidney cells, or could be released promptly after synthesis. Further work clearly is necessary to characterize the origin and hormonal mechanism(s) that influence U-EGF both in developing and adult animals.

Differences in the IR-EGF concentration of SMG between males and females observed on day 26 in the present study (Fig. $4 A$ ) are consistent with an earlier report of the appearance of convoluted granular tubules in male SMG by day 25 (14). Thus both biochemical and morphological sexual dimorphism in SMG seem to develop at the same time. In contrast, kidneys, which also exhibit a number of sexual dimorphisms in the adult (25), showed no sex difference in relative weight or IR-EGF concentrations on day 26 (Fig. $4 B$ ). In adult animals the relative weights of both SMG and kidneys were clearly higher in males than females. The $\mathrm{M}>\mathrm{F}$ sex difference in SMG-IR-EGF was maintained in the adult animals (Fig. $4 A$ ); however, the sex differences in adult kidney and urine IR-EGF levels (Fig. $4 B$ and $C$ ) were opposite in direction to the sex difference $(M>F)$ in kidney weight, SMG weight, and SMG EGF concentration. Also, adult female kidney responded to testosterone treatment by growth but not by an increase in IR-EGF concentration (Fig. 5). These results indicate that the major determinant of the biochemical and morphological sexual dimorphism of SMG in the adult mouse is testosterone. The sexual dimorphisms of kidney are more complex. Testosterone may account for the $\mathrm{M}>\mathrm{F}$ kidney weight, but the mechanism(s) for the $F>M$ kidney and urine EGF dimorphisms remains to be explained. 

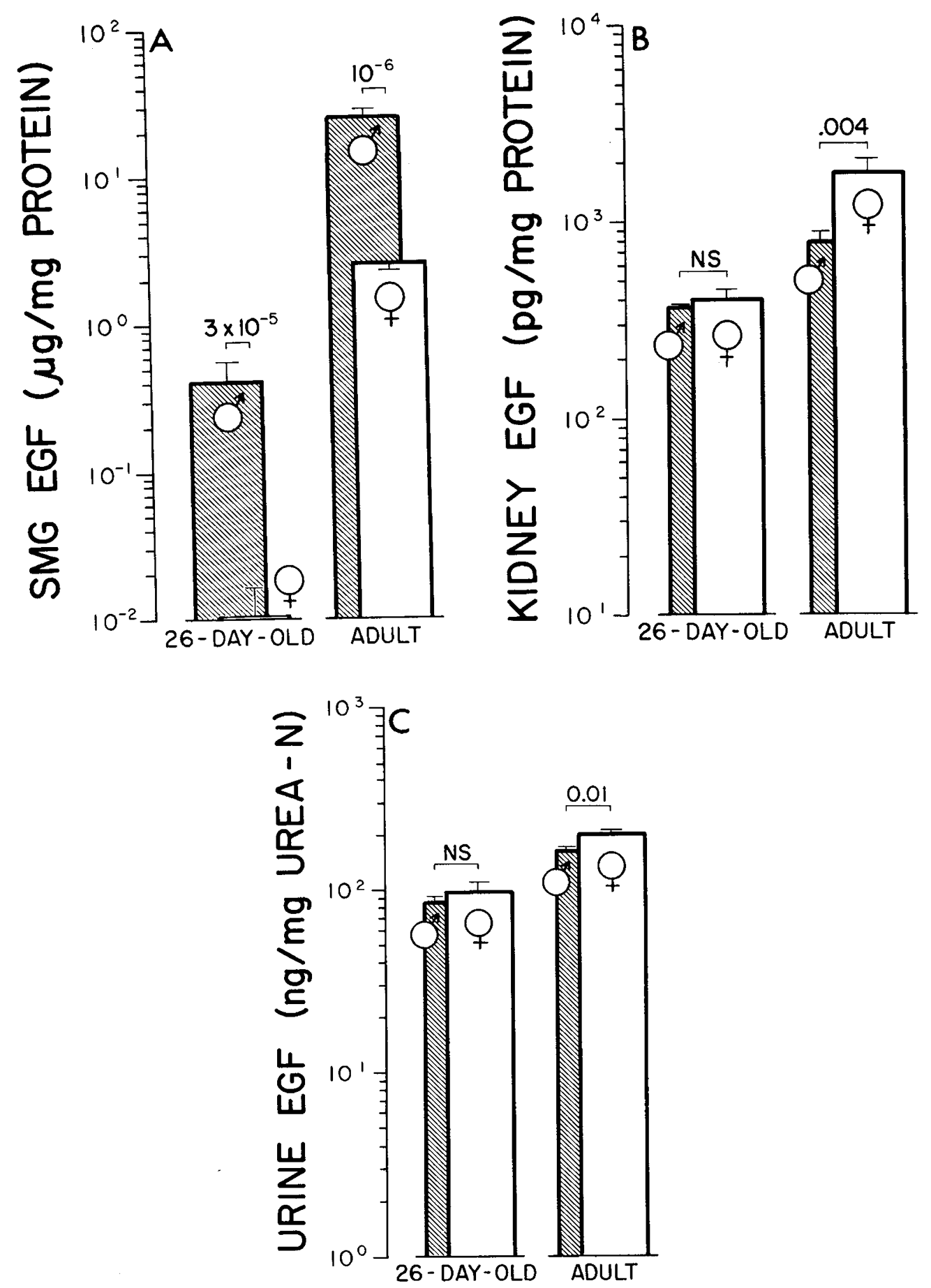

Fig. 4. Relative immunoreactive EGF concentrations (mean $\pm \mathrm{SE}$ ) in male (shaded columns) and female (open columns) 26-day-old and adult mice: $A$, submandibular gland; $B$ kidneys; and $C$ urine. $p$ values for the differences are given above the columns. 


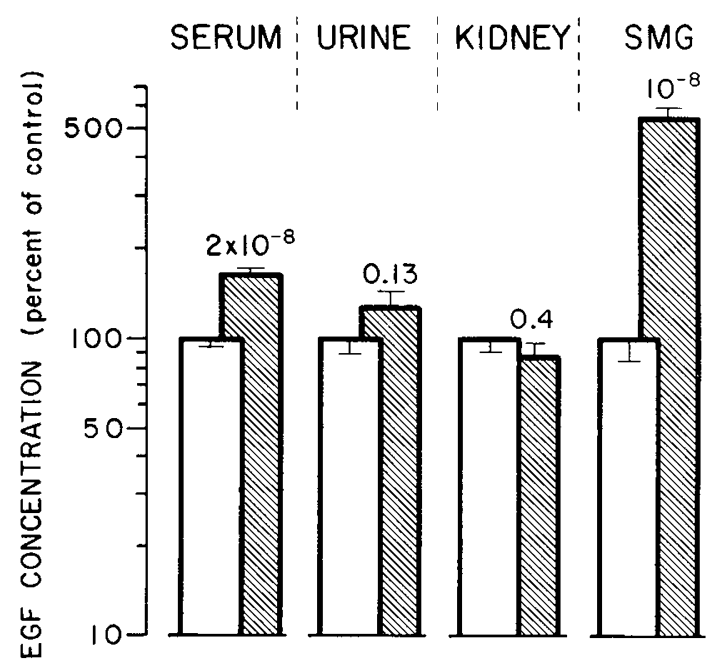

Fig. 5. Immunoreactive EGF concentrations (mean $\pm \mathrm{SE}$ ) in serum, urine, kidneys, and SMG in testosterone-treated adult female mice ( shaded columns) relative to controls (open columns, mean $=100$ ). $p$ values for the differences are given above the columns.

Acknowledgments. The authors thank Ms. Thelma Macaso and Ms. Hye Kim for skillful technical assistance and Ms. Sharon Schuler and Ms. Dawn Bostrom for typing the manuscript.

\section{REFERENCES}

1. Savage CR Jr, Inagami T, Cohen S 1972 The primary structure of epidermal growth factor. J Biol Chem 247:7612-7621

2. Gospodarowicz D, Moran JS 1976 Growth factors in mammalian cells culture. Ann Rev Biochem 45:531-558

3. Cohen S 1962 Isolation of a mouse submaxillary gland protein accelerating incisor eruption and eyelid opening in newborn animals. J Biol Chem 237:1555-1562

4. Tarkington RW, Males JL, Cohen S 1971 Synthesis and storage of epithelialepidermal growth factor in submaxillary gland. Cancer Res 31:252-256

5. Van Noorden S, Heitz P, Gregory H, Polak JM, Pearse AG 1977 Immunostaining of mouse epithelial-epidermal growth factor. Improved light and electron microscopical localization. Gastroenterology 72:826 (abstr)

6. Byyny RL, Orth DN, Dohen S, Doyne ES 1974 Epidermal growth factor. Effects of androgens and adrenergic agents. Endocrinology 95:776-782

7. Gresik EW, Schenkein I, Van der Noen H, Barka T 1981 Hormonal regulation of epidermal growth factor and protease in the submandibular gland of the adult mouse. Endocrinology 109:924-929

8. Walker P, Weichsel ME Jr, Hoath S, Poland SE, Fisher DA 1981 Effect of thyroxine, testosterone and corticosterone on nerve growth factor (NGF) and epidermal growth factor (EGF) concentration in adult female mouse submaxillary gland. Dissociation of NGF and EGF responses. Endocrinology 109:582-587

9. Byyny RL, Orth DA, Cohen S 1972 Radioimmunoassay of epidermal growth factor. Endocrinology 90:1261-1266

10. Frati L, Cenci G, Sbaraglia Teti DV, Covelli I 1976 Levels of epidermal growth factor in mice tissues measured by a specific radioreceptor assay. Life Sci 18:905-912

11. Hirata Y, Orth DN 1979 Concentrations of epidermal growth factor, nerve growth factor and submandibular gland renin in male and female mouse tissue and fluids. Endocrinology 105:1382-1387

12. Perheentupa J, Lakshmanan J, Hoath SB, Kim H, Fisher DA 1983 Hormonal modulation of epidermal growth factor concentration in mouse serum and bile. The Endocrine Society, 65th Annual Meeting, San Antonio, TX, abstr 79

13. Scott J, Urdea M, Quiroga M, Sanchez-Pescador R, Fong N, Selby M, Rutter WJ, Bell GI 1983 Structure of a mouse submaxillary messenger RNA encoding epidermal growth factor and seven related proteins. Science 221:236-239

14. Chretien M 1977 Action of testosterone on the differentiation and secretory activity of a target organ. The submaxillary gland of the mouse. Int Rev Cytol 50:333-396

15. Lowry OH, Rosebrough NJ, Farr AL, Randall RJ 1951 Protein measurement with Folin phenol reagent. J Biol Chem 193:265-275

16. Henry RJ 1967 Clinical Chemistry: Principles and Technics. Medical Division, Harper and Row Publishers, New York, pp. 266-270

17. Hoath S, Lakshmanan J, Scott SM, Fisher DA 1983 Effect of thyroid hormones on epidermal growth factor concentration in neonatal mouse skin. Endocrinology 112:308-314

18. Perheentupa J, Lakshmanan J, Fisher DA 1984 Epidermal growth factor in neonatal mouse urine. Maturative effect of thyroxine. Pediatr Res 18:10801084

19. Savage CR Jr, Cohen S 1972 Epidermal growth factor and new derivative. Rapid isolation procedures and biological and chemical characterization. $\mathrm{J}$ Biol Chem 247:7609-7611

20. Walker P, Weichsel ME Jr, Eveleth D, Fisher DA 1982 Ontogenesis of nerve growth factor and epidermal growth factor in submaxillary glands and nerve growth factor in brains of immature mice. Correlation with ontogenesis of serum levels of thyroid hormones. Pediatr Res 16:520-524

21. Perheentupa J, Lakshmanan J, Macaso T, Fisher DA 1984 Growth hormone increases neonatal mouse urine epidermal growth factor. Acta Endocrinol (Copenh) 105:184-187

22. Gonzales F, Lakshmanan J, Hoath S, Fisher DA 1984 Effect of oestradiol-17B on uterine epidermal growth factor concentration in immature mice. Acta Endocrinol (Copenh) 105:425-428

23. Dailey GE, Kraus JN, Orth DN 1978 Homologous radioimmunoassay for human epidermal growth factor (urogastrone). J Clin Endocrinol Metab 46:929-936

24. Coghlan JP, Niall HD, Penschow J, Tregear GW 1984 Hybridization histochemistry: tissue localization of gene expression. In: Abstracts of the 7th International Congress of Endocrinology, July 1-7, 1984. Quebec City, Canada. Excerpta Medica International Congress series 652, Amsterdam, abstr P3, p 5

25. Dofuku R, Tettenborn U, Ohno S 1971 Testosterone "regulon" in the mouse kidney. Nature New Biol 232:5-7 